\title{
ATUAÇÃO DO ENFERMEIRO NA GESTÃO DE RECURSOS MATERIAIS NA ATENÇÃO PRIMÁRIA À SAÚDE
}

\section{THE NURSE'S ACTIVITIES IN THE MANAGEMENT OF MATERIAL RESOURCES IN PRIMARY HEALTH CARE}

\section{Jéssica de Jesus Ferreira ${ }^{1} *$ Beatriz Francisco Farah $^{2} *$ Herica Silva Dutra $^{3} *$ Maria Tereza Ramos Bahia $^{4}$ * Nádia Fontoura Sanhudo $5 *$ Meirieli Faza Franco 6 \\ RESUMO}

Objetivo: compreender como enfermeiros supervisores da Atenção Primária à Saúde realizam a gestão dos recursos materiais. Método: abordagem qualitativa, tipo estudo de caso, com 13 enfermeiros supervisores e/ou assistenciais, que responderam a entrevista semiestruturada. Resultados: a gestão dos recursos materiais foi entendida de maneira diversificada pelos enfermeiros mencionando etapas isoladas de previsão e provisão; controle, organização e gestão dos recursos materiais; sendo realizadas de forma incompleta. É parametrizada pelo nível central sem prever o estoque de segurança e dividida em duas etapas: previsão e controle de estoque. Fatores desafiadores: estrutura física das unidades, interrupções frequentes no intervalo entre a solicitação e a entrega dos materiais. Potencialidades: integração da equipe multidisciplinar, comunicação entre os supervisores por meio de redes sociais. Conclusão: a racionalização e a gestão dos recursos materiais eficiente e eficaz são necessárias, pois contribuirão para manutenção da continuidade da assistência, garantindo a qualidade.

Palavras-chave: Recursos Materiais em Saúde; Gestão de Materiais; Atenção Primária à Saúde; Enfermagem; Ensino.

\section{ABSTRACT}

Objective: To understand how nurses supervising Primary Health Care manage material resources. Method: a qualitative case study research, with 13 supervising and / or assisting nurses, who answered the semi-structured interview. Results: the management of material resources was understood in a diversified way by nurses mentioning isolated stages of forecasting and provision; control, organization and management of material resources; being performed incompletely. It is parameterized by the central level without forecasting safety stock and is divided into two stages: forecast and stock control. Challenging factors: physical structure of units, frequent interruptions in the interval between ordering and delivery of materials. Potentialities: integration of multidisciplinary team, communication between supervisors through social networks. Conclusion: Rationalization and efficient and effective material resource management are necessary as they will contribute to the continuity of care, ensuring quality.

Keywords: Material Resources in Health; Materials Management; Primary Health Care; Nursing; Teaching.

\footnotetext{
${ }^{1}$ Enfermeira, residente em intensivismo, urgência e emergência do Programa Multiprofissional em Saúde do Hospital Maternidade Therezinha de Jesus da Faculdade de Ciências Médicas de Juiz de Fora, Juiz de Fora, Minas Gerais (MG), Brasil. (32)99174-2838, jessicafelixs@gmail.com. ORCID: https://orcid.org/0000-0003-2444-264X

${ }^{2}$ Doutora em Saúde Coletiva, Docente do Departamento de Enfermagem Básica da Faculdade de Enfermagem da Universidade Federal de Juiz de Fora, Juiz de Fora, Minas Gerais (MG), Brasil. ORCID: https://orcid.org//0000-0002-3345-0601

${ }^{3}$ Doutora em Ciências da Saúde pela Universidade Estadual de Campinas, docente Adjunta da Faculdade de Enfermagem e docente permanente do Programa de Pós-Graduação Mestrado em Enfermagem da Universidade Federal de Juiz de Fora, Juiz de Fora, Minas Gerais (MG), Brasil. ORCID: https://orcid.org//0000-0003-2338-3043

${ }^{4}$ Mestre em enfermagem, Docente do Departamento de Enfermagem Básica da Faculdade de Enfermagem da Universidade Federal de Juiz de Fora, Juiz de Fora, Minas Gerais (MG), Brasil. ORCID: https://orcid.org/0000-0002-6254-114X

${ }^{5}$ Doutora em enfermagem, Docente do Departamento de Enfermagem Básica da Faculdade de Enfermagem da Universidade Federal de Juiz de Fora, Juiz de Fora, Minas Gerais (MG), Brasil. ORCID: https://orcid.org/0000-0001-9714-2854

${ }^{6}$ Enfermeira, Mestranda do Programa de Pós-Graduação em enfermagem da Universidade Federal de Juiz de Fora, Juiz de Fora, Minas Gerais (MG), Brasil. ORCID: https://orcid.org/0000-0001-5041-4787
} 


\section{INTRODUÇÃO}

Os custos nos serviços de saúde têm produzido um desequilíbrio nas contas, devido à grande demanda por serviços de saúde e pouca oferta ${ }^{(1)}$. Incorporação de tecnologias e expansão das especialidades médicas e serviços têm elevado o valor despendido na saúde. Acrescido a estes fatores têm-se o envelhecimento populacional, aumentando a demanda por cuidados de saúde ${ }^{(2)}$, associado a carência de profissionais qualificados, ausência de sistemas de controle de custos e desperdícios a nível assistencial ${ }^{(1)}$. Recursos humanos e medicamentos representam o maior custo gerado pelos serviços de saúde, seguidos pelos recursos materiais e equipamento, sendo que os materiais de consumo contribuem com $30 \%$ a $40 \%$ dos recursos necessários para a assistência à saúde ${ }^{(3)}$. O gerenciamento de custos nas organizações de saúde tem por finalidade a redução de gastos, envolvendo a área administrativa e os responsáveis por cada unidade, buscando integrar o conhecimento dos diversos profissionais para a criação, efetivação e manutenção desse sistema ${ }^{(3)}$.

A gestão de recursos materiais (GRM) propõe a otimização dos recursos por meio do gerenciamento dos processos, desde a aquisição dos materiais até o controle de estoque. Para uma gestão efetiva, eficiente e eficaz devem-se associar aspectos de qualidade e segurança ao uso racional dos recursos e redução dos desperdícios ${ }^{(4)}$.
Com o envolvimento do enfermeiro na gestão e gerenciamento de custos e a existência de uma lacuna na produção científica, verifica-se a necessidade de investimento em pesquisas e atualização na área $^{(5)}$. A enfermagem atua no gerenciamento de custos visando alcançar os objetivos da instituição e atender as necessidades daqueles que buscam o serviço ${ }^{(4)}$.

A GRM tem sido desenvolvida por meio de processos constituídos de etapas contínuas e dinâmicas $^{(4)}$, contemplando as seguintes atividades desenvolvidas: programação, que compreende as etapas de padronização, classificação, especificação e previsão; compra, abrangendo os processos de controle de qualidade e licitação; recepção; armazenamento dos materiais; distribuição e controle.

A etapa de previsão e provisão nos serviços de saúde nem sempre é realizada conforme evidências científicas. Consiste em levantar as necessidades, identificando a quantidade e a especificidade de materiais para suprir a demanda específica de cada setor. A cota mensal (CM) é calculada pela expressão matemática $\mathrm{CM}=\mathrm{CMM}+\mathrm{ES}^{(4)}$. O consumo médio mensal (CMM) deve ser avaliado pelo período mínimo de três meses somando o estoque de segurança (ES).

O estoque de segurança é calculado pela fórmula $\mathrm{ES}=10$ a $20 \%$ do $\mathrm{CMM}+\mathrm{CTR}$. Para o cálculo do consumo diário por tempo de reposição (CTR) faz-se a divisão do CMM 
por 30 dias e multiplica-se pelo número de dias de espera pela reposição $(\mathrm{N})^{(4)}$.

A realização da etapa de previsão e provisão sem critérios ou adoção parcial da fórmula tem comprometido o funcionamento dos serviços e a assistência aos usuários. Acredita-se que a realização de todos as etapas pelo enfermeiro supervisor viabilize uma GRM eficiente com otimização dos recursos, evitando desperdícios, favorecendo uma assistência contínua e de qualidade ${ }^{(4)}$.

Na Atenção Primária à Saúde (APS) os enfermeiros supervisores de Unidades Básicas de Saúde (UBS) são responsáveis por realizar as atividades assistenciais e administrativas, sendo a GRM uma delas ${ }^{(6)}$. Na maioria das vezes, a GRM é limitada à provisão de insumos e redução de custos, negligenciando a averiguação da qualidade dos mesmos ${ }^{(7)}$.

A vivência das pesquisadoras na APS permitiu observar a dificuldade que os supervisores das UBS demonstravam com relação a GRM incluindo o armazenamento, administração da diversidade e peculiaridade dos materiais utilizados, bem como fatores limitadores enfrentados pela gestão da APS para a aquisição e distribuição destes ${ }^{(7)}$.

Diante do exposto surgiu a necessidade de refletir como estes materiais têm sido gerenciados na APS pelos enfermeiros supervisores, sua percepção sobre como realizam esta atividade e quais fatores potencializam e limitam sua atuação na GRM.
Traçou-se como objetivo compreender como os enfermeiros supervisores da APS realizam a GRM e quais são os fatores limitadores e potencializadores para o desenvolvimento da mesma.

\section{MÉTODOS}

Pesquisa de abordagem qualitativa, tipo estudo de caso.

O cenário do estudo foi um município do estado de Minas Gerais, Brasil. Este município possui $63 \mathrm{UBS}$, das quais 41 tem implantada a Estratégia Saúde da Família (ESF) e 22 o modelo tradicional de assistência à saúde. Dessas UBS, 30 têm o enfermeiro como supervisor.

Os participantes foram enfermeiros selecionados a partir da listagem do nível central de UBS, por contato telefônico. Após o aceite de participar da pesquisa, foi agendada entrevista individual na UBS do participante, conforme a disponibilidade de cada profissional.

Foram incluídos 13 enfermeiros que acumulavam as funções de supervisão e assistência por no mínimo seis meses. Desses, 11 trabalhavam em UBS com ESF e duas no modelo tradicional.

Os critérios de exclusão foram aqueles que não puderam ser entrevistados no dia marcado pela pesquisadora em virtude de problemas pessoais ou do trabalho. 
Para a coleta de dados utilizou-se entrevista semiestruturada. As questões norteadoras foram: como vem sendo realizada a GRM pelos enfermeiros nas UBS? Os enfermeiros vêm realizando a previsão e provisão de recursos materiais utilizando qual cálculo? As unidades estão providas de recursos materiais necessários para atender as necessidades do serviço e da população?

Os dados foram coletados entre setembro a outubro de 2017, de forma individual, no próprio local de trabalho do participante, em ambiente tranquilo e privativo. As entrevistas foram gravadas em áudio, e transcritas na íntegra totalizando 239 minutos e 35 segundos.

A fim de preservar o anonimato dos entrevistados foi atribuído código utilizandose a letra "E" acrescida dos números sequenciais arábicos de E1 a E13.

A análise dos dados foi realizada por meio da Análise de Conteúdo, do tipo Análise Temática. $^{8}$

A pesquisa foi encaminhada ao Comitê de Ética em Pesquisa com Seres
Humanos da Universidade Federal de Juiz de Fora e aprovada com parecer de $\mathrm{n}^{\mathrm{0}} 1.874 .665$ de 04/07/17.

\section{RESULTADOS}

Foi possível observar a predominância do sexo feminino $(84,6 \%)$ entre os participantes. A idade variou entre 31 e 58 anos, o tempo de atuação profissional entre oito e 32 anos. Todos possuíam pósgraduação, com experiência profissional na APS entre dois e 32 anos e como supervisor de seis meses e 11 anos.

Emergiram dos resultados três categorias empíricas: Percepção dos Enfermeiros da Atenção Primária à Saúde sobre Gestão de Recursos Materiais; Realizando a Gestão de Recursos Materiais na Atenção Primária à Saúde; As Potencialidades e Desafios da Gestão de Recursos Materiais na Atenção Primária à Saúde (Quadro 1).

Quadro 1- Síntese das categorias e núcleos de sentido. Juiz de Fora, MG, Brasil, 2017

\begin{tabular}{|c|c|c|c|c|}
\hline \multicolumn{3}{|c|}{ Categorias } & Núcleos de sentido & Falas dos enfermeiros \\
\hline \multirow{2}{*}{ 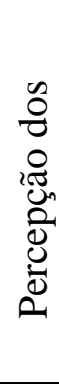 } & \multirow{2}{*}{ 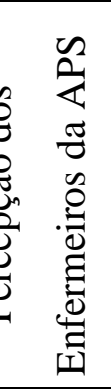 } & \multirow{2}{*}{$\begin{array}{l}\sum_{0} \\
0 \\
0 \\
0 \\
0 \\
0\end{array}$} & $\begin{array}{l}\text { Previsão e provisão de } \\
\text { materiais } \quad \text { visando } \\
\text { redução de custo }\end{array}$ & $\begin{array}{l}\text { Pela minha experiência [...], administrar } \\
\text { esses recursos seria criar uma metodologia, } \\
\text { de saber quanto gasta por mês e quanto } \\
\text { pede, questão de validade e não ter perdas. } \\
\text { (E8) }\end{array}$ \\
\hline & & & Controle de materiais & $\begin{array}{l}\hat{E} \text { controlar a quantidade que você tem de } \\
\text { necessidade para distribuir para a }\end{array}$ \\
\hline
\end{tabular}




\begin{tabular}{|c|c|c|}
\hline & & $\begin{array}{l}\text { população e ao mesmo tempo, controlar a } \\
\text { perda e desperdícios. (E2) }\end{array}$ \\
\hline & $\begin{array}{ll}\text { Organização } & \text { dos } \\
\text { recursos materiais } & \end{array}$ & $\begin{array}{l}\text { Eu entendo que é um recurso que a gente } \\
\text { tem para tentar organizar as demandas, em } \\
\text { cima daquilo que a gente solicita, para que } \\
\text { não haja desperdício nem falta de materiais. } \\
\text { (E11) }\end{array}$ \\
\hline & $\begin{array}{lr}\text { Gestão de } & \text { recursos } \\
\text { materiais } & \text { como } \\
\text { processo } & \\
\end{array}$ & $\begin{array}{l}\text { É planejar de acordo com suas necessidades } \\
\text { e estar acompanhando o uso e avaliando a } \\
\text { saída. Estar atento a validade e } \\
\text { conservação de cada produto. A gente não } \\
\text { participa da parte de aquisição de } \\
\text { materiais, o que representa um problema. Só } \\
\text { fazemos a solicitação para a secretaria de } \\
\text { saúde. (E10) }\end{array}$ \\
\hline \multirow{2}{*}{ 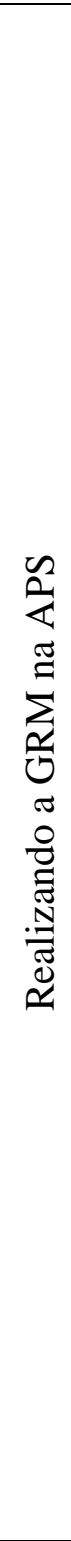 } & $\begin{array}{l}\text { Previsão dos recursos } \\
\text { materiais }\end{array}$ & $\begin{array}{l}\text { A gente olha o que tem no estoque e pede o } \\
\text { complemento. (E2) } \\
\text { Então a gente solicita material uma vez por } \\
\text { mês. É, mais pelo conhecimento do dia a dia } \\
\text { mesmo, da gente saber o que sai mais e o } \\
\text { que não sai [...]. Faço isso mais pelas } \\
\text { experiências do dia a dia mesmo. (E1) } \\
\text { Eu tenho que prever material para } 40 \text { dias. } \\
\text { (E4) } \\
\text { A gente não pede com estoque, pede o que } \\
\text { gasta em uma média de até } 60 \text { dias. (E8) } \\
\text { Sempre colocamos o quantitativo de } 20 \% \text { em } \\
\text { cima do que é pedido, mas tem coisas que eu } \\
\text { confesso para você, eu jogo para cima. (E5) }\end{array}$ \\
\hline & Controle de estoque & $\begin{array}{l}\text { Então o controle de estoque é muito } \\
\text { importante, então a economia que } \\
\text { representa você controlar e usar } \\
\text { adequadamente faz uma diferença. (E2) } \\
\text { Então a gente, equipe de enfermagem, criou } \\
\text { uma planilha de saída de insumos, de cada } \\
\text { material a gente vai registrando, data da } \\
\text { saída e a quantidade que a gente tirou [...]. } \\
\text { Centralizar mais esse almoxarifado, facilita } \\
\text { o controle. (E3) } \\
\text { Eu utilizo planilhas que eu tenho. Eu mesma } \\
\text { que criei. (E4) } \\
\text { [...] comecei a ter problemas de sumiço de } \\
\text { alguns materiais [...]. Eu que recebo e faço } \\
\text { o armazenamento de todo material e a } \\
\text { dispensação. A chave fica comigo e eu tenho } \\
\text { um controle maior do que entra e sai. (E13) }\end{array}$ \\
\hline
\end{tabular}




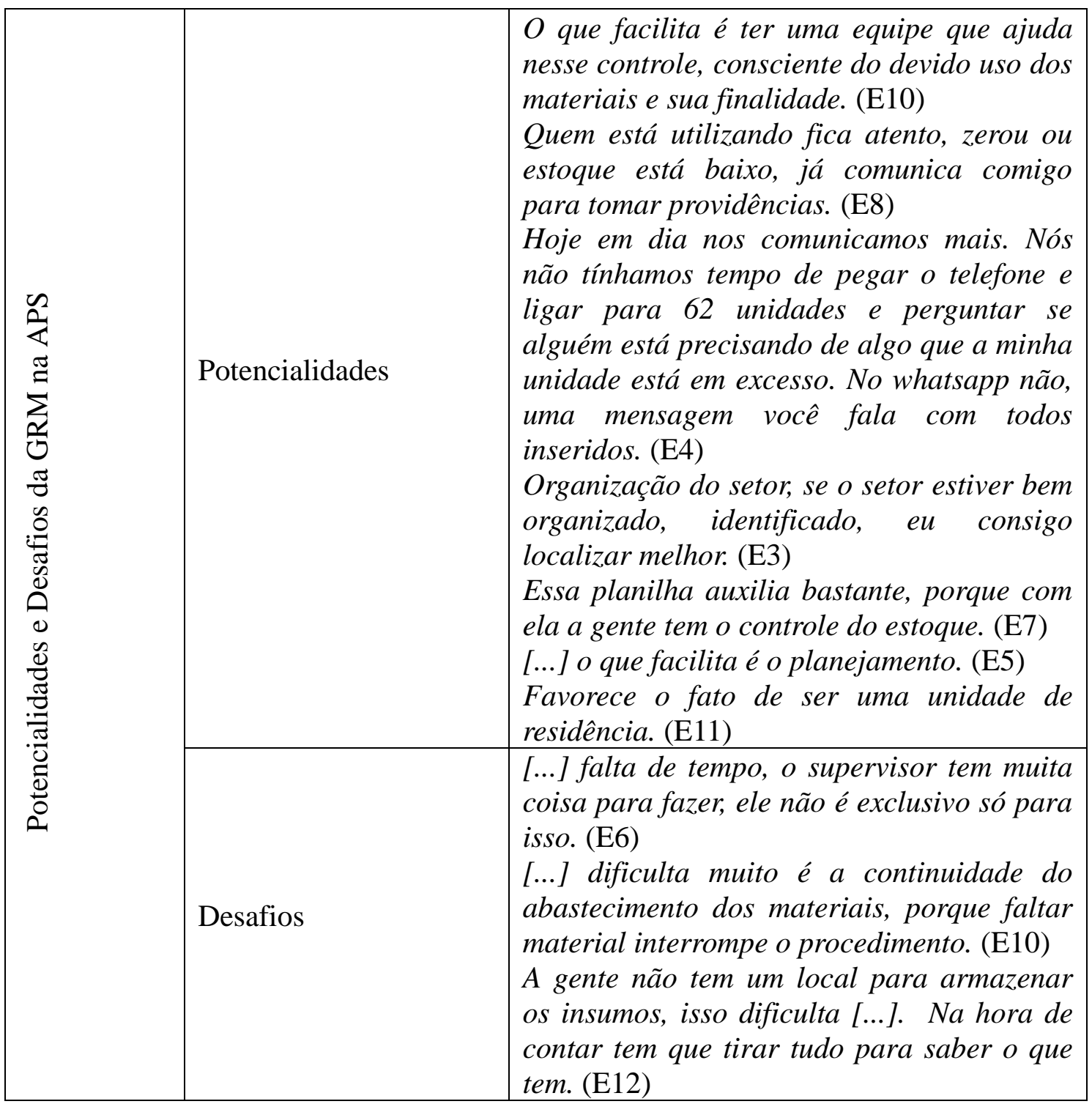

Fonte: Os autores.

\section{DISCUSSÃO}

\section{Percepção dos enfermeiros sobre gestão de} recursos materiais

A GRM é apresentada como um método racional e científico, capaz de nortear os processos de trabalho. Permite a solução de problemas e dificuldades administrativas, mediante a organização da unidade; aplicação de programas, controle de fluxo e assistência e utilização de recursos de forma sistematizada ${ }^{(10)}$.

A GRM foi entendida de maneira diversificada. Alguns enfermeiros descreveram-na como etapa de previsão e provisão; uns citaram o controle, e outros a organização e gestão desses recursos como processo. A previsão foi considerada o cerne desta atividade. Por meio dela os enfermeiros realizavam a estimativa do quantitativo de materiais necessário para determinado 
período, requisitando o essencial para que não houvesse falta nem acúmulo. Os enfermeiros demonstraram a preocupação com o uso adequado do dinheiro público e o não desperdício de materiais que acarretaria no aumento de $\operatorname{gastos}^{(10)}$.

A GRM está inserida nas competências e responsabilidades do trabalho do enfermeiro ${ }^{(11)}$. É o profissional que possui no currículo conteúdos de administração e gerenciamento, que o capacita para desempenhar a GRM. As diretrizes curriculares nacionais incluem a competência administração/gerenciamento como requisito para a formação de todos os profissionais de saúde $^{(12)}$. Porém, evidências questionam sobre a lacuna de conhecimento específicos sobre os custos em saúde na formação do enfermeiro $^{(13)}$. Acredita-se ser essa temática fundamental na formação do enfermeiro ${ }^{(14)}$, mantendo-a posteriormente por meio de educação permanente.

Para o funcionamento dos serviços de saúde se faz necessário o estoque de materiais, entretanto, a ausência de controle deste eleva o custo dos serviços prestados ${ }^{(14)}$. O cálculo adequado da previsão de materiais permite uma melhor acomodação dos estoques, reduzindo o comprometimento de recursos, viabilizando a continuidade da assistência prestada nos serviços de saúde ${ }^{(13)}$.

Grandes estoques ocasionam perdas comprometendo as verbas disponibilizadas para o setor ${ }^{(3,13)}$. Ambas situações puderam ser verificadas na prática de enfermeiros da APS.

$\mathrm{O}$ enfermeiro possui entre suas responsabilidades a contenção de gastos ${ }^{(11,14-}$ 15), realizada por meio de uma efetiva supervisão e controle do emprego dos materiais de consumo e permanentes.

$\mathrm{O}$ controle dos recursos materiais produz informações necessárias para previsão, reduz o extravio e contribui para o uso racional dos insumos ${ }^{(16)}$. Os enfermeiros entenderam que GRM é controlar os insumos e, que a ausência desta função sobre os recursos materiais pode desencadear desperdícios e aumento de custos.

A organização foi mencionada como fundamental para GRM, propiciando melhor funcionamento da unidade e da prestação da assistência. A organização é uma atividade complexa, que envolve pessoas, tecnologias, materiais e recursos necessários para o alcance dos objetivos institucionais. Tal prática na GRM gera melhor acessibilidade aos insumos, fácil alcance, manejo do quantitativo e controle de validade ${ }^{(3)}$. Apesar dos esforços dos supervisores, percebeu-se a dificuldade de realizar plenamente a organização dos materiais.

A GRM foi entendida também como um processo. Foram mencionadas algumas etapas da GRM, como planejamento, previsão, compra e armazenamento. A atuação do enfermeiro supervisor de UBS não tem sido contemplada na etapa de aquisição 
dos materiais, comprometendo o atendimento de requisitos técnicos e qualidade desejada dos mesmos para assistência segura.

Destaca-se a importância da realização de avaliação dos materiais por meio de parecer técnico a fim de garantir a qualidade dos materiais empregados na assistência e contribuir para o controle dos $\operatorname{custos}^{(7)}$. O enfermeiro é apto a participar dos processos de compra de materiais, por ser o profissional que administra justificando a necessidade de sua integração nesse processo ${ }^{(10)}$.

Portanto, cabe aos enfermeiros se organizarem e se capacitarem para aprimorar a GRM na APS.

Realizando a gestão recursos materiais na Atenção Primária à Saúde

A percepção da GRM pelos enfermeiros contemplava duas etapas: previsão e controle de estoque.

A previsão e o controle de estoque baseavam-se no perfil de consumo/tempo ${ }^{(15)}$. A realização da avaliação da integridade e validade e a contagem dos produtos do estoque tinham como objetivo quantificar insumos necessários para o próximo período.

$\mathrm{O}$ pedido de materiais era feito mensalmente, utilizando-se de planilha impressa ou virtual encaminhada ao nível central. A reposição dos insumos era de 30 dias, período adequado para o atendimento das unidades e capacidade de armazenamento dos insumos. Isso contribui para diminuição de investimentos em estoque e custos com manutenção ${ }^{(15)}$.

A contagem de estoque foi citada como ponto de partida para a realização do novo pedido de materiais. A solicitação de materiais não era realizada de maneira uniforme nas unidades, com períodos de 30 até 60 dias. Os pedidos eram baseados em conhecimento dos enfermeiros relativos aos gastos de materiais da unidade ou por meio do cálculo parametrizado orientado pelo nível central.

A parametrização foi definida pelo nível central a partir do ano de 2015. O cálculo consistia em subtrair da $\mathrm{CM}$ o estoque existente (EE), gerando a solicitação para o próximo mês.

Verificou-se a não inclusão do estoque de segurança, podendo ocasionar insuficiência de recursos materiais necessários ao funcionamento da unidade ${ }^{(4)}$.

As variações na realização da previsão dos recursos materiais podem gerar acúmulo de insumos nas unidades. Estratégia justificada para garantir o suprimento em períodos de falta. A insegurança quanto a entrega dos insumos e a imprevisibilidade da demanda influenciavam na tomada de decisão. Outros problemas comumente enfrentados incluíam: a interrupção do envio de insumos às UBS por falta no estoque central, problemas de infraestrutura e na compra de produtos, dentre outros. 
$\mathrm{O}$ estudo do comportamento da demanda permite a criação de modelos para previsão, favorecendo a utilização de um modelo matemático apropriado ao perfil das unidades, além de facilitar o dimensionamento do estoque suplementar ${ }^{(17)}$. Portanto, cabe ao nível central avaliar e adequar a proposta de previsão. Recomendase considerar as particularidades de cada UBS e os determinantes sociais na programação e planejamento da assistência em saúde ${ }^{(12)}$.

O controle de estoque foi citado como fundamental para a GRM, pois gerava informações que orientavam a necessidade do novo pedido ou compra do próximo mês, favorecendo a continuidade da assistência e a redução dos custos quando realizado adequadamente.

Para facilitar o acompanhamento do consumo de materiais e a previsão de insumos, os enfermeiros criaram planilhas específicas não padronizadas para registrar a entrada e saída de materiais. Contudo, verificou-se a necessidade de padronização destas e do número de dias para reposição.

As estratégias de tecnologias da informação nos serviços de saúde são necessárias para otimizar o desempenho organizacional, quando focam apenas os processos administrativos para fins de cobrança e controle repercutindo negativamente na qualidade dos serviços $\operatorname{prestados}^{(18)}$.
A falta de informatização nas unidades dificultava a manutenção do controle do estoque. Os registros de entrada e saída eram realizados manualmente, sendo necessário ao final de 30 dias recontar todo o estoque. $\mathrm{O}$ controle obtido pelo uso do sistema informatizado, adaptado às necessidades da instituição de saúde auxilia efetivamente nos processos logísticos, permitindo maior organização, controle revertido em melhoria para o serviço, profissionais e pacientes ${ }^{(18)}$.

Outro aspecto considerado no controle dos materiais era a precariedade das instalações das UBS, levando a uma improvisação dos locais de armazenamento dos insumos, dificultando o acompanhamento das fichas de prateleiras.

É necessário que haja um almoxarifado nas unidades de saúde para a guarda dos materiais, garantindo a integridade dos insumos e gerenciamento de entrada e saída $^{(19)}$. cabendo ao supervisor comunicar a gestão da APS sobre a inadequação desse local de guarda, para definirem a melhor estratégia e solucionar o problema.

Os enfermeiros relataram experiências de desaparecimento e uso indevido de materiais. Afim de solucionar estes problemas, alguns profissionais limitaram o acesso de pessoas ao almoxarifado. Porém essa decisão pode comprometer o desenvolvimento das atividades da equipe de saúde. 
Verificou-se a importância de fazer uma administração participativa, pois estimula o compromisso, responsabilidade do uso e controle consciente dos materiais. Assim, a GRM deixa de ser apenas uma preocupação quantitativa para incorporar dimensões como a imprevisibilidade da demanda, estrutura física e recursos humanos, exigindo conhecimento e reavaliação constante desses processos ${ }^{(5)}$.

\section{As potencialidades e desafios da gestão recursos materiais na atenção primária à saúde}

As ações cotidianas do enfermeiro são influenciadas pelas várias características intrínsecas e extrínsecas do sistema de saúde, dentre as quais citam-se a disponibilidade, as limitações e a qualidade dos materiais e instrumentos disponíveis nos serviços de saúde ${ }^{(20)}$. Ressalta-se a importância da capacitação dos profissionais para a realização da GRM.

Vários aspectos foram pontuados que favoreciam a realização da GRM em suas unidades, dentre eles o trabalho em equipe. $\mathrm{O}$ bom relacionamento interpessoal na equipe contribui para um atendimento integral aos usuários, a compreensão de todo o processo de trabalho e a divisão de tarefas. A GRM não é função exclusiva do supervisor da unidade, mas uma atividade compartilhada, e sua eficácia depende da colaboração de toda equipe.
Outro aspecto considerado essencial para a GRM foi a comunicação efetiva entre os colegas de trabalho, que dividem informações pertinentes ao processo de controle de estoque e consumo. A comunicação nos serviços é reflexo da necessidade das trocas de informações, criando interdependência e parceria ${ }^{(21)}$.

A comunicação entre os supervisores e equipe gestora da APS tem sido realizada por meio digital, a fim de diminuir o temporesposta sobre solicitações de materiais. O uso das redes sociais nos serviços de saúde ampliou a interação, através de grupos abertos ou fechados, facilitando a troca de informações e experiências ${ }^{(22)}$. Os enfermeiros supervisores utilizavam desta ferramenta trocando informações para diminuir o desperdício, perda de material, quando necessitavam de algum recurso inexistente ou insuficiente na UBS que atuavam. Verificou-se o empenho dos enfermeiros de liderar e reunir esforços na busca de parcerias e recursos aumentando a abrangência e o grau de resolução.

$\mathrm{O}$ número suficiente de profissionais atuantes nas UBS foi indicado como um fator contribuinte para a operacionalização da GRM, assim como a presença de estagiários e residentes $^{(23)}$.

Os recursos humanos são o cerne da capacidade produtiva, eles representam a maior parte dos investimentos disponibilizados ao atendimento da 
população ${ }^{(24)}$. No entanto, quando estes recursos se encontram deficitários nas UBS a organização do trabalho é prejudicada e atividades são negligenciadas, gerando frustração nos profissionais, que não alcançam a qualidade de atendimento que gostariam.

Dentre os desafios da GRM, foi apontada a grande demanda de atividades por assumirem a atribuição de supervisor da unidade associada à função de enfermeiro da equipe, ocasionando sobrecarga de trabalho ${ }^{(5)}$.

Outra questão foi a existência da interrupção no fornecimento de materiais para as UBS. A equipe de enfermagem tem vivenciado dificuldades diante das necessidades dos usuários e da exigência de produtividade da gestão. Essa situação expõe a unidade e equipe. $\mathrm{O}$ supervisor deve refletir com a equipe e gestão da APS sobre as causas dessa insuficiência e os limites impostos ao desenvolvimento das atividades profissionais.

A estrutura física foi apontada como fator de forte influência sobre a GRM, pois a existência de local específico para a guarda de materiais facilita o controle de estoque e garante a preservação da integridade dos produtos. Entretanto, quando esta não é adequada, o armazenamento e controle dos materiais sofrem prejuízos ${ }^{(3)}$.

Para a superação dos desafios, foi ressaltado a importância da educação permanente, a fim de realizar a GRM de forma correta e eficiente. A educação permanente vem sendo proposta pelo SUS como uma importante ferramenta para a resolução de problemas no processo de trabalho das equipes ${ }^{(25)}$.

Portanto, é imprescindível que enfermeiros e gestão repensem condutas da GRM, pois influenciará na melhoria da distribuição dos investimentos, na utilização eficiente e eficaz dos recursos financeiros e materiais nos serviços públicos.

\section{CONSIDERAÇÕES FINAIS}

Os enfermeiros compreenderam a GRM de forma diversificada, mencionando etapas isoladas de previsão e provisão; controle, organização e gestão dos recursos materiais; sendo realizadas de forma incompleta. Foi identificada preocupação com o controle dos custos e uso racional dos recursos. Entretanto, os enfermeiros não participavam da avaliação da qualidade e aquisição dos recursos materiais. Negligenciar alguma das etapas prejudica a GRM, gera maiores custos, na compra de materiais que as vezes não atenderá as necessidades das UBS.

Como fatores desafiadores relacionaram a estrutura física das unidades, o intervalo entre a solicitação e a entrega dos materiais, a não adoção do estoque de segurança na parametrização proposta pela gestão, a escassez de tempo para a realização da GRM diante da sobrecarga de trabalho. A falta de padronização da solicitação e controle dos estoques conduziu os enfermeiros a 
realizarem a GRM de forma empírica, individual e particularizada. Apesar dos fatores desfavoráveis os enfermeiros vêm apresentando e desenvolvendo estratégias pontuais, construindo instrumentos que os auxiliam na previsão dos materiais, evitando o desabastecimento das UBS e a desassistência ao cidadão.

A integração da equipe multidisciplinar e a comunicação entre os supervisores por meio de redes sociais foram fatores potencializadores para a GRM na APS.

Sugere-se investimentos na educação permanente dos supervisores para melhoria da GRM, a inclusão do enfermeiro nas etapas de avaliação da qualidade e aquisição de materiais, a elaboração de planilhas de controle de insumos, parametrização que contemple a fórmula de cálculo de materiais e as diversidades das UBS. Há necessidade de um sistema informatizado que integre as unidades e facilite o controle interno, pois contribuirá para o acompanhamento fidedigno e atual do estoque, diminuindo o tempo dispensado na contagem dos materiais.

Como limitações desta investigação aponta-se o desenho qualitativo que explora as percepções dos participantes e as particularidades do contexto no qual estão inseridos. Entretanto, acredita-se que este estudo contribui para alavancar a discussão sobre a GRM na APS.

\section{REFERÊNCIAS}

1. Oliveira AG, Silva CL, Blanski MBS. Gestão de custos como instrumento de governança pública. Criterio Libre [Internet]. 2016; [acesso em 22 de abril de 2019];24(1):57-87. Disponível em: https://revistas.unilibre.edu.co/index.php/crite riolibre/article/view/72/55

2. Bush M. Addressing the Root Cause Rising Health Care Costs and Social Determinants of Health. N. C. med. j. 2018;79(1):26-9. Disponível em: https://doi.org/10.18043/ncm.79.1.26

3. Melo AB, Gomes BRS, Pinheiro BDSB, Martins LFJ, Palheta MG, Santos RSU et al. A gestão de materiais médico-hospitalar em hospital público. Rev. Gest. Saúde [Internet]. 2016 [acesso em 14 de julho de 2019];65(2):339-46. Disponível em: https://dialnet.unirioja.es/servlet/articulo?codi go=5555865

4. Castilho V, Gonçalves VLM. Gerenciamento de recursos materiais. In: Gerenciamento em enfermagem. Rio de Janeiro: Guanabara Koogan; 2016.

5. Vieira GCG, Bento LA. Gestão de custos hospitalares: o conhecimento do enfermeiro. Rev. Uningá [Internet]. 2016 [acesso em 28 de junho de 2019];48(1):56-60. Disponível em:

http://revista.uninga.br/index.php/uninga/artic le/view/1284/904

6. Farah BF, Dutra HS, Sanhudo NF, Costa LM. Percepção de enfermeiros supervisores sobre liderança na atenção primária. Rev. Cuid. [Internet]. 2017 [acesso em 25 de novembro de 2019];8(2):163855. Disponível em:

http://dx.doi.org/10.15649/cuidarte.v8i2.398.

7. Silva RNA, Lima AKM, Filha FSSC, Vilanova JM, Silva FL. Conhecimento e entendimento de enfermeiros sobre as ações gerenciais na atenção primária à saúde. Ciên. Saúde [Internet]. 2016 [acesso em 25 de janeiro de 2020];9(1):21-9. Disponível em: 
http://dx.doi.org/10.15448/1983-

$\underline{652 X .2016 .1 .21028}$

8. Bardin L. Análise de conteúdo. Lisboa: Edições 70; 2016.

9. Machado MH, Vieira ALS, Oliveira E. Construindo o perfil da enfermagem. Enferm. Foco [Internet]. 2012 [acesso em 14 de dezembro de 2019]; 3(3):119-122. Disponível em:

http://enfermagematualizada.com/UserFiles/F ile/Artigo/ARTIGO_REVISTA_COFEN.pdf

10. Bogo PC, Bernadino E, Castilho V, Cruz EDA. O enfermeiro no gerenciamento de materiais em hospitais de ensino. Rev. Esc. Enferm. USP [Internet]. 2015 [acesso em 23 de agosto de 2019]; 49(4):0632-9. Disponível em: $\quad$ http://dx.doi.org/10.1590/S0080623420150000400014

11. Dubois CA, Damour D, Brault I, Dallaire C, Déry J, Duhoux A et al. Which priority indicators to use to evaluate nursing care performance? A discussion paper. J. adv. nurs. 2017 [cited 2020 fev 02];73(12):3154-67. Available from: https://doi.org/10.1111/jan.13373

12. Brasil. Ministério da Saúde. Portaria $n^{\circ}$ 198, de 13 de fevereiro de 2004. Institui a Política Nacional de Educação Permanente em Saúde como estratégia do Sistema Único de Saúde para a formação e o desenvolvimento de trabalhadores para o setor e dá outras providências. Diário Oficial da União 16 fev 2004. Disponível em: https://www.nescon.medicina.ufmg.br/bibliot eca/imagem/1832.pdf

13. Souza SESM, Silva AR. Contribuição do enfermeiro no processo de gerenciamento de custo nas instituições hospitalares. Rev. GEHSH. [Internet]. 2017 [acesso em 30 de outubro de 2019];8(2):338-62. Disponível em: https://periodicos.unb.br/index.php/rgs/article/ view/3732

14. Gunawan J, Aungsuroch Y. Managerial competence of first-line nurse managers: A concept analysis. Int. J nur. pract [Internet]. 2017 [cited 2019 set 10];23(1):12502.
Available

from:

https://doi.org/10.1111/ijn.12502

15. Carvalho BG, Peduzzi M, Nunes EFPA, Leite FS, Silva JAM. Gerência de unidade básica de saúde em municípios de diferentes portes: perfil e instrumentos gerenciais utilizados. Rev. Esc. Enferm. [Internet]. 2017 [acesso em 21 de maio de 2019];48(5):90714.

Disponível

em:

http://dx.doi.org/10.1590/S0080-

$\underline{6234201400005000018}$

16. Gonçalves PS. Administração de materiais. Rio de Janeiro: Elsevier; 2013.

17. Raimundo EA, Dias CN, Guerra $\mathrm{M}$. Logística de medicamentos e materiais em um hospital público do Distrito Federal. RAHIS [Internet]. 2015 [acesso em 05 de maio de 2019];12(2):61-9. Disponível em: https://doi.org/10.21450/rahis.v12i2.2384

18. Alami H, Lehoux P, Gagnon MP, Fortin JP, Fleet R, Ahmed MAA. Rethinking the electronic health record through the quadruple aim: time to align its value with the health system. BMC med. inform. decis. mak. [Internet]. 2020 [cited 2020 jan 28];20(1):1-5. Available

from:

https://doi.org/10.1186/s12911-020-1048-9

19. Fernandes JC, Cordeiro BC. O gerenciamento de Unidades Básicas de Saúde no olhar dos enfermeiros gerentes. Rev. Enferm. UFPE [Internet]. 2018 [acesso 15 de novembro de 2019];12(1):194-202. Disponível em: https://doi.org/10.5205/19818963-v12i01a23311p194-202-2018.

20. Andrade LDF, Simões ACM, Henriques AHB, Sousa ATO, Pinto MB. Desempenho do enfermeiro em suas atividades laborais na Atenção Primária à Saúde. Rev. Enferm. Atenção Saúde [Internet]. 2016 [acesso 21 de novembro de 2019];5(1):51-63. Disponível em: https://doi.org/10.18554/reas.v5i1.1749

21. Henderson M, Dahnke MD. The ethical use of social media in nursing practice. MedSurg nurs. [Internet]. 2015 
[cited 2019 jul 13]; 24(1):62-5. Available from:

https://search.proquest.com/openview/87d7f4 22e9033f6af1d346f82a029748/1?pqorigsite $=$ gscholar $\& \mathrm{cbl}=30764$

22. Lima SGP, Cassiano ACM, Vermelho SCSD. A utilização de redes sociais digitais na área da saúde: uma revisão sistemática. Saúde e pesquisa [Internet]. 2015 [acesso em 26 de março de 2019];8(0):93-101. Disponível em: https://periodicos.unicesumar.edu.br/index.ph p/saudpesq/article/view/3775/2515

23. Soder RM, Santos LE, Oliveira IC et al. Práticas de enfermeiros na gestão do cuidado na atenção básica. Rev. cuba. enferm. 2020; 36(1): 2815 . Available from: http://revenfermeria.sld.cu/index.php/en f/article/view/2815.

24. Cordeiro ALAO, Fernandes JD, Mauricio MDALLD, Silva RMO, Barros CSMA, Romano CMC. Capital humano na gestão das enfermeiras em hospitais. Rev. Esc. Enferm. USP [Internet]. 2017 [acesso em 06 de maio de 2019]; 51:e03232. Disponível em: http://dx.doi.org/10.1590/s1980$\underline{220 \times 2016030203232}$

25. Arnemann CT, Lavich, CRP, Terra MG, Mello AL, Raddatz M. Educação em saúde e educação permanente: ações que integram o processo educativo da enfermagem. Rev. baiana enferm. [Internet]. 2018 [acesso em 14 de junho de 2019];32:e24719. Disponível em: http://dx.doi.org/10.18471/rbe.v32.24719

\section{Autor correspondente}

Meirieli Faza Franco. Rua dos Canários, no 80, Caeté, Juiz de Fora - Minas Gerais (MG). CEP: 36102-000. (32)99840-4441. meiri_faza@hotmail.com.

Submissão: 2021-08-25

Aprovado: 2021-09-07 